\title{
Simulation of Control Algorithm for Active Reduction of Transversal Vibrations of Beams by Piezoelectric Elements Based on Identification of Bending Moment
}

\author{
M.S. KOZIEN ${ }^{a, *}$ AND Ł. ŚCISŁO ${ }^{b}$ \\ ${ }^{a}$ Cracow University of Technology, Faculty of Mechanical Engineering, Institute of Applied Mechanics, \\ Al. Jana Pawła II 37, 31-864 Kraków \\ ${ }^{b}$ Cracow University of Technology, Faculty of Electrical and Computer Engineering, \\ Department of Automatic Control \& IT, Warszawska 24, 30-155 Kraków
}

\begin{abstract}
Application of piezoelectric elements to active reduction of bending vibrations of the beams is known well in the literature. During vibrations, an external excitation may vary in the time domain, including its form in the frequency domain. It should have influence on their response. The problem of proper selection of the control parameter in the control algorithm used to reduce vibrations arises. In the article, simulations of a control algorithm based on detection of bending moment are analytically tested. The solution for the transient type of vibrations are obtained by the finite difference method (FDM). Analysis for two separated natural modes was performed. The analysis shows the possibility to design a control algorithm based on detection of the bending moment.
\end{abstract}

DOI: 10.12693/APhysPolA.128.A-56

PACS: 02.30.Jr, 43.40.Ar, 43.40.Cw, 45.20.da

\section{Introduction}

Response of actual engineering structures may have sometimes a multimodal-type form in the frequency domain. Examples of such structures include car suspension (bimodal) [1] or the HORN system focusing particles by means of the magnetic field (multimodal) [2,3].

Reduction of bending vibrations of beams by piezoelectric elements is a well-known idea. Such reduction can be realized in a passive or active form for different types of the beam response (unimodal, bimodal, or multimodal) [4-20].

In practical reduction of vibrations, one of the key issues is the optimal placement of piezoelements on the beam. The general rule provides for placing the elements in areas where the bending moment has maximum values $[7,19,20]$. This idea was applied in $[4,5]$ to simulation of reduction of torsional vibrations of the beam.

In an attempt to design the reduction control algorithm for the multimodal case, the separated single-mode type of beam vibrations was taken under consideration. The simulations were performed for the simply-supported steel beam. One pair of piezoelectric elements, mounted to the beam for vibration reduction was modeled afterwards $[10,11]$ by two concentrated bending moments applied to the beam in the places where the piezoelectric elements ended. The control algorithm was based on the idea of applying a voltage to piezoelectric elements. The value of the voltage was proportional to the curvature of line of beam deformation, i.e., in practice, to the

*corresponding author; e-mail: kozien@mech.pk.edu.pl internal bending moment. The cases of the single-modal beam response for the two different separated modes of vibrations are analyzed in this article. Excitation of vibrations were done by the application of harmonically variable in time point force acting in arbitrary chosen cross-section with different frequencies connected to values of the two natural frequencies of a beam. Simulations were performed by solving the problem by the finite difference method (FDM).

The aim of the study presented in this paper was to verify the control algorithm for reducing the bending vibrations of the beam for separated modes, based on the directly detected bending moment in the region of the maximum curvatures for considered mode.

\section{Reduction of vibrations by piezoelectric elements}

\subsection{Acting of piezoelectric elements}

When considering the Bernoulli-Euler bending vibrations of the beam, the following equation of forced vibrations can be used:

$$
\begin{gathered}
E J_{y} \frac{\partial^{4} w(x, t)}{\partial x^{4}}+\rho A \frac{\partial^{2} w(x, t)}{\partial t^{2}}= \\
q(x, t)+\sum_{i=1}^{N} F_{i}(t) \delta\left(x-x_{i}\right)+\sum_{j=1}^{M} M_{j}(t) \frac{\partial \delta\left(x-x_{i}\right)}{\partial x},
\end{gathered}
$$

where $w(x, t)$ is the transverse displacement of the beam, $E$ is the Young modulus of the material, $\rho$ is the volume density of the material, $J_{y}$ is the moment of inertia of the beam cross-section, $A$ is the area of the beam cross-section, $L$ is length of beam, $M_{j}(t)$ is the $j$-th concentrated moment, $x_{j}$ is the point of an action of the $j$-th moment, $F_{i}(t)$ is the $i$-th concentrated force, $x_{i}$ is 
the point of an action of the $i$-th concentrated force, $q(x, t)$ is the distributed force, and $\delta(x)$ is the Dirac delta distribution [21].

According to the Bernoulli-Euler theory, transversal displacements of the beam during bending vibrations produce the so-called bending moment $M_{g}(x, t)$. The dependence between the deflection and the bending moment is given by equation (2). The curvature of the beam $\kappa(x, t)$, which is connected to its deformation, is proportional to the bending moment. What is more, the value of the moment is directly linked to the transverse displacement of the beam's neutral line $w(x, t)$ through either a close or a linear relationship (3). It follows from equations that the moment of zero value is connected to zero curvature and zero strain displacement. It can therefore be concluded that from the theoretical point of view, the problem of the active vibration reduction in beams using piezoelectric elements comes down to the creation of a counter-bending moment (anti-bending moment) $M_{g}^{*}(x, t)$ which, theoretically, in each section of the beam at any moment of time gives the total moment equal to zero when added to $M_{g}(x, t)(4)$.

$$
\begin{aligned}
& M_{g}(x, t)=E J_{y} \kappa(x, t), \\
& \kappa(x, t)=-\frac{\frac{\partial^{2} w(x, t)}{\partial x^{2}}}{\sqrt{1+\left(\frac{\partial w(x, t)}{\partial x}\right)^{\frac{3}{2}}}} \approx-\frac{\partial^{2} w(x, t)}{\partial x^{2}}, \\
& M_{g}(x, t)+M_{g}^{*}(x, t)=0 .
\end{aligned}
$$

However, the following restrictions apply while the vibration reduction using piezoelectric elements is considered in a real case:

- It is practically impossible to generate a countermoment function as a continuous function of the spatial variable $x$.

- Determination of the current bending moment $M_{g}(x, t)$ function does not take place in an indirect way, which is a source of error and delay.

- The impact of the piezoelectric elements is discrete and concerns a specific area (length of the element).

- There is an inertia in the system which should be taken into account when considering the control algorithm.

- There may be an issue of the stability of the control system.

Let us consider a symmetric configuration of the attached identical piezoelectric elements (symmetrical on both external surfaces of the beam and driven $\pi$ out of phase with the same voltage signal) in a wafer arrangement, i.e. when the piezoelectric element is very long alongside beam, compared to its thickness it is polarized through [10]. Moreover, distribution of the electric charge is constant on the surface of the piezoelectric element that is attached to the beam. The action of the pair of piezoelectric actuators can be expressed in the form of two equivalent bending moments which have the same value but opposite signs and act on the opposite ends of the element (see Fig. 1). The value of the generated

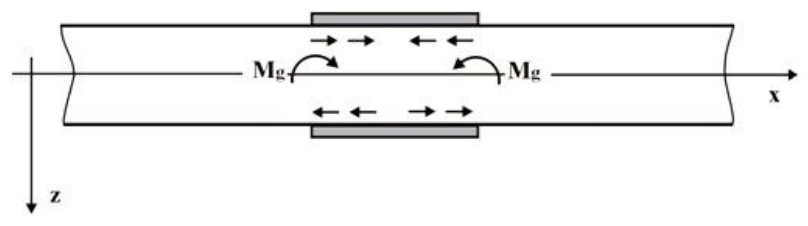

Fig. 1. Bending moments generated by the piezoelectric element glued to the beam.

equivalent bending moment $M_{e q}$ can be written in the form (5), where $E_{p}$ is the Young modulus of the piezoelectric material, $h$ and $h_{p}$ are thicknesses of the beam and the piezoelectric element, respectively, and $d_{z x}$ is the charge constant of the piezoelectric material [10]. Hence, the value of the equivalent bending moment depends on the type of the materials that the beam and the attached element are made out of, the thickness of the beam, as well as on thickness of the element and the applied voltage. Finally, the action of the piezoelectric actuators in the equation of motion of the beam (1) can be described in the form (6), where $x_{0}$ is the center coordinate of the element, $L_{p}$ is its length, and $h_{p}$ is its thickness:

$$
\begin{aligned}
& M_{e q}(t)=\frac{3}{2} E J_{y} \frac{E_{p}\left[\left(h+h_{p}\right)^{2}-h^{2}\right]}{E_{p}\left[\left(h+h_{p}\right)^{3}-h^{3}\right]+E h^{3}} \frac{d_{z x}}{h_{p}} V(t), \\
& M_{g}^{*}\left(x_{0}, t\right)= \\
& M_{e q}(t)\left[\frac{\partial \delta\left(x_{0}+0.5 L_{p}\right)}{\partial x}-\frac{\partial \delta\left(x_{0}-0.5 L_{p}\right)}{\partial x}\right] .
\end{aligned}
$$

From mechanical point of view it is convenient for a theoretical analysis that, after application of voltage to the piezoelectric elements, two counter-bending moments that are distant from each other by a distance equal to the length of the element are created, when taking into consideration the equation of vibration of the beam (1) and practical implementation of that method. But the fundamental question arises about the number of elements required for the realization of the process and their location.

In the case of the beam vibration reduction for a particular form of vibration (unimodal response), the theoretical analysis shows that the piezoelectric element for the so-called quasi-optimum position should be located in a place (or one of the places) with the greatest curvature resulting from the concerned vibration mode type. Theoretically, one element located in the region with maximum curvature is enough [19, 20], but for modes with several regions with maximum curvatures, the problem of number of actuators and their placement can be still discussed [7]. For the Bernoulli-Euler beam model, the curvature of the beam in a particular section is associated with the value of the bending moment shown in 
equation (2). This means that a piezoelectric element or elements should be applied in the place/places with the highest vibration curvature or otherwise, the largest value of the bending moments understood as the generalized inner strength. Thus, in theoretical papers, the optimal placement of unimodal quasi-optimum piezoelectric elements is determined. The problem consists in determining the number of elements (theoretically, one for the mode would be sufficient), and above all, the amplitude of the applied voltage that is substantially different for different modes.

\subsection{Selection of the voltage value. Control algorithm}

According to the general formula, it can be assumed that the voltage applied to the element generates proportional bending moments at the edges (linear description). In accordance with the method discussed above, the generated moments are counter-moments with respect to the ones that occur when the beam is vibrating and crosscutting forces are generated. Control algorithms that adopt voltages as control variables can be found in the literature $[13,14,17]$. In their papers, the authors considered the concept of applying, to each of the piezoelectric elements, a voltage (variable in time) which was proportional to the value of the bending moment at a defined instant of time and located in the beam crosssection in which the element was attached. The coefficient of proportionality between the voltage and a chosen parameter connected with bending moments (curvature, linear strain, a set of transversal displacements) must be matched to the system and depend on the method used to identify the value of the bending moment. This can be determined on-line, e.g. based on:

- Measuring the voltage on the piezoelectric element that is acting as a sensor and located on the outer surface of the beam, opposite to that on which a piezoelectric element acting as an activator is situated (unsymmetrical configuration of the piezoelectric elements [10]).

- Finding the bending moment from the differential formula of the finite difference method, in its simplest form shown in equation (7). The method is particularly convenient when performed simulations using the finite element method, because the structure is divided into nodes that are distant from each other by given value of the $\Delta x$ :

$$
\begin{aligned}
& M_{g}^{*}(x, t)=M_{g}(x, t) \approx \\
& -E J_{y} \frac{w(x-\Delta x, t)-2 w(x, t)+w(x+\Delta x, t)}{\Delta x}= \\
& k_{F B} \frac{w(x-\Delta x, t)-2 w(x, t)+w(x+\Delta x, t)}{\Delta x} .
\end{aligned}
$$

- The $\varepsilon_{x}$ linear deformation is measured on the outer surface of the beam using a electrofusion extensometer or a lath of strain gauges and with the use of formula (8), where $z_{\max }$ is the distance from the neutral axis of bending to the outer surface of the beam on which a strain gauge is attached:

$$
\begin{aligned}
& M_{g}^{*}(x, t)=M_{g}(x, t)=\frac{E J_{y} \varepsilon_{x}(x, t)}{z_{\max }}= \\
& k_{E X} \varepsilon_{X}(x, t) .
\end{aligned}
$$

A smart beam with PZT elements can be defined as the structure that, by using a control system, can sense external disturbance and respond to it actively using a designed control algorithm so as to maintain its dynamics within the desired limits. Such structures comprise distributed active devices like sensors and actuators that are attached to the structure. The simplest control algorithm that can be implemented to suppress the occurring vibrations in the system is the direct feedback of the output parameter back into the system. The control algorithm for the discussed case is presented in Fig. 2.

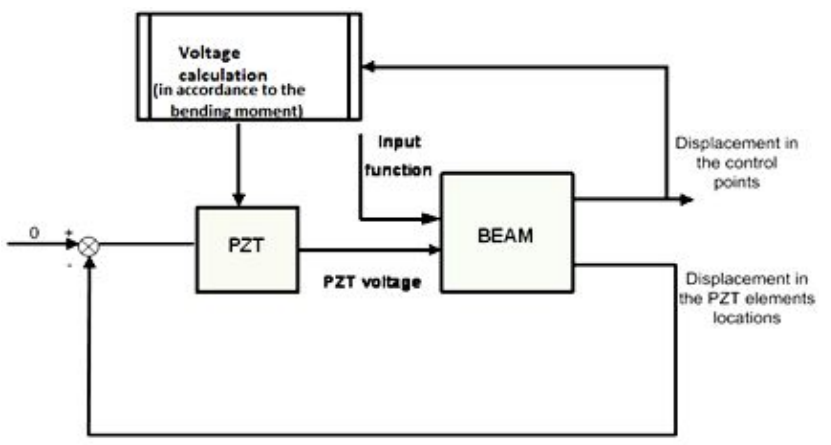

Fig. 2. A block diagram of the feedback control system.

The sensor generates a voltage when the structure is vibrating. The voltage is proportional to the bending moment of the structure and the signal is fed back into actuator using a control algorithm, as shown in Fig. 2. The actuating voltage under a control algorithm can be determined based on formula (5). It produces the antibending moment for reducing the vibration of the system (6). For obtaining the dynamic response under a given external loading condition, a modal analysis can be used and the nodal displacement can be calculated. The voltage is calculated according to the value of nodal displacements in the previous time step.

\section{Analytical simulation}

The simulation was performed for the reduction of forced vibrations on the beam that was simply supported at both ends. The external loading was assumed in form of a harmonic concentrated force $F_{1}(t)=F_{0}(\sin (v t))$, where $\nu$ is the excitation angular frequency, applied at the center of the beam $\left(x_{1}=0.5 L\right)$. Analyses were performed for the beam made of steel $(E=210$ [GPa], 
$\left.\rho=7800\left[\mathrm{~kg} / \mathrm{m}^{3}\right]\right)$, with length $L=0.3[\mathrm{~m}]$, and rectangular cross-section of witdh $b=0.01[\mathrm{~m}]$ and thickness $h=0.002[\mathrm{~m}]$. The force amplitude was $F_{0}=1[\mathrm{~N}]$. Two cases of vibration were taken under consideration for simulation of the control algorithm:

- the frequency $\nu$ of force applied to the beam was close to the first natural frequency of the beam $\left(\omega_{1}=328.5[\mathrm{~Hz}]\right)$;

- the frequency $\nu$ of force applied to the beam was close to the third natural frequency of the beam $\left(\omega_{3}=2957[\mathrm{~Hz}]\right)$.

For both cases, the maximum curvature of the deformed vibrating beam falls in the middle of the beam, therefore a piezoelectric element positioned this way should made possible and be sufficient to reduce the excited vibrations.

The vibration equation modeling the feedback control system, with the internal damping of the beams material neglected and including a model of the piezoelectric elements as actuators for reducing the excited vibrations, has the form $(9)$, where $M_{g}^{*}(x, t)$ is the anti-bending moment proportional to actual bending moments occurring in points where the piezoelectric elements are placed and $\Delta t$ is a time delay between the actual time and the time of detection of the anti-bending moment with respect to formula (7). Two cases of detecting the bending modes are tested:

- at the ends of the piezoelectric element $\left(x=x_{1} \pm\right.$ $\Delta x)$,

- in the middle of the piezoelectric element $\left(x=x_{1}\right)$.

$$
\begin{aligned}
E & J_{y} \frac{\partial^{4} w(x, t)}{\partial x^{4}}+\rho A \frac{\partial^{2} w(x, t)}{\partial t^{2}}=F_{0} \sin (\nu t) \delta(x-0.5 L) \\
& +M_{g}^{*}(0.5 L-\Delta x, t-\Delta t) \frac{\partial \delta(x-0.5 L+\Delta x)}{\partial x} \\
& -M_{g}^{*}(0.5 L+\Delta x, t-\Delta t) \frac{\partial \delta(x-0.5 L-\Delta x}{\partial x} .
\end{aligned}
$$

Simulations were performed by finding numerical response of the structure by using the finite difference method. The solution has a form of the transient-type analysis which can model real-life implementation of the control algorithm. Due to the applied method of finding the solution, the concentrated anti-bending moments were replaced by a set of concentrated anti-forces applied to three neighboring nodes resulting from finite difference discretization, with values given by

$$
F^{*}(x, t)=\frac{M_{g}^{*}(x, t)}{\Delta x}
$$

and modeling the whole area of piezoelectric element (Fig. 3). Finally, the problem of control of vibration reduction can be described by

$$
\begin{aligned}
E & J_{y} \frac{\partial^{4} w(x, t)}{\partial x^{4}}+\rho A \frac{\partial^{2} w(x, t)}{\partial t^{2}}=F_{0} \sin (\nu t) \delta(x-0.5 L) \\
& +F^{*}(0.5 L+\Delta x, t-\Delta t) \delta(x-0.5 L-\Delta x)
\end{aligned}
$$

$$
\begin{aligned}
& -2 F^{*}(0.5 L, t-\Delta t) \delta(x-0,5 L) \\
& +F^{*}(0.5 L-\Delta x, t-\Delta t) \delta(x-0.5 L+\Delta x) .
\end{aligned}
$$

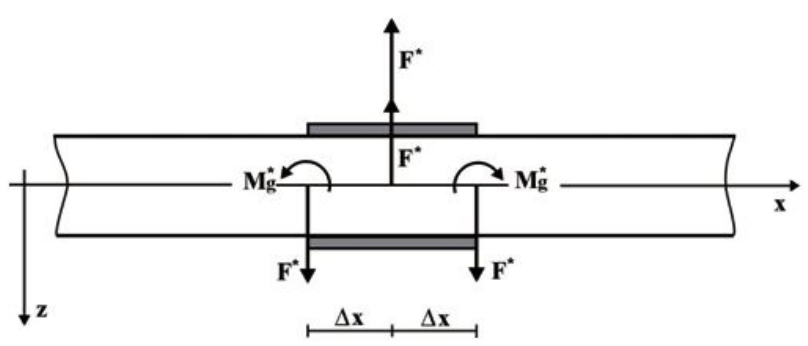

Fig. 3. Replacing a pair of concentrated moments attached to ends of a piezoelectric element by two pairs of concentrated forces.

The central difference scheme with error of the second order based on three nodes in the time domain was applied to replace the second derivative in the time domain. The central difference with error of the second order was applied to replace the fourth derivative in the space domain. The boundary conditions of the simply-supported beams in the analytical form (12) were transformed into the finite difference form by the same central difference scheme as for the internal nodes by inserting the imaginary nodes and applying the defined boundary conditions in the formulas. For all analyzed cases, the equation is solved by dividing the beam length into ten sub-domains $\left(\Delta x=\frac{L}{10}\right)$, and the time integration domain into steps of $1 / 1000$ of period of the first natural mode.

$$
\left\{\begin{array}{l}
w(0, t)=0, \\
\frac{\partial^{2} w(0, t)}{\partial x^{2}}=0, \\
w(L, t)=0, \\
\frac{\partial^{2} w(L, t)}{\partial x^{2}}=0 .
\end{array}\right.
$$

Due to application of the finite difference method, the investigated natural frequencies were a little different then theoretical ones, $\omega_{1}=328.5[\mathrm{~Hz}]$ and $\omega_{3}=$ $2745[\mathrm{~Hz}]$. Transversal displacements of the middle of beam without application of the control algorithm are shown in Figs. 4 and 5 for excitation frequencies equal to natural frequencies of the first and the third mode, respectively. For the sake of legibility of visualization of the results obtained only for a limited time range domain $(t \in[0,0.01][\mathrm{s}])$, the simulations are shown in the following figures. The resonance type of beam response can be observed.

Reduction of vibration after application of the control algorithm was observed for the two analyzed cases of bending moment identification (on the ends or in the middle of the piezoelectric element). The feedback coefficients for these cases were different for different modes and moment identification methods, however the finally determined values of concentrated anti-forces were almost the same for the same modes. The detailed values are given in Table, and the example of response of 


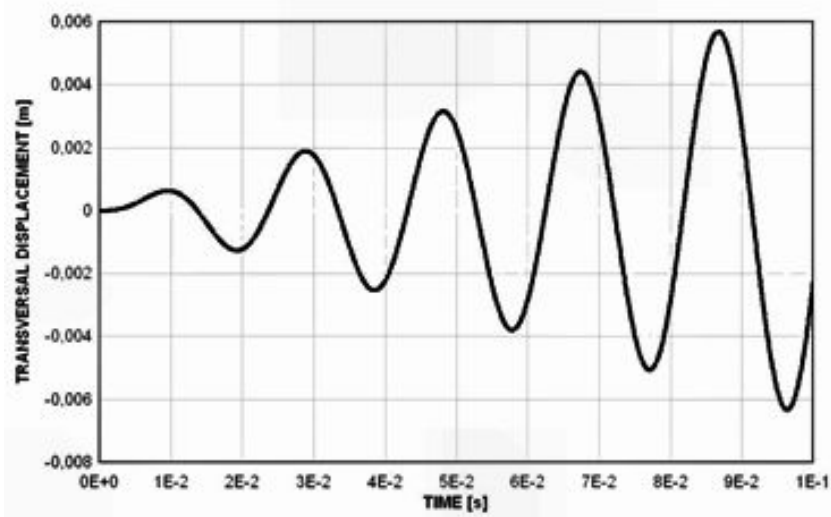

Fig. 4. Resonance response in the middle of the beam for the first natural mode.

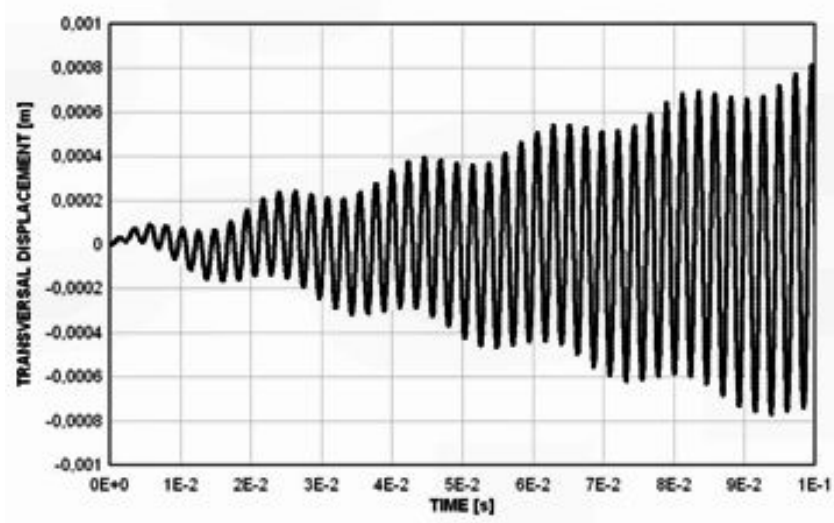

Fig. 5. Resonance response in the middle of the beam for the third natural mode.

the same point and the same analyzed period of time as in Fig. 4 and Fig. 5 are shown in Figs. 6-8. The range of the simulation was assumed to be $1[\mathrm{~s}]$, and the maximum value of displacement $\max _{t}[w(0.5 L, t)]$ given in Table denotes the maximum absolute value of transversal displacement in the middle of the beam in the analyzed period of time.

TABLE

Results of reduction of transversal vibrations of the middle of beam

\begin{tabular}{c|c|c|c}
\hline $\begin{array}{c}\text { Position } \\
\text { of bending moment } \\
\text { identification }\end{array}$ & $\begin{array}{c}\text { Feedback } \\
\text { coefficient } \\
k_{F B}\end{array}$ & $\begin{array}{c}\text { Type } \\
\text { of action }\end{array}$ & $\begin{array}{c}\text { Maximum } \\
\text { displacement }_{\max _{t}[w(0.5 L, t)]}\end{array}$ \\
\hline \multicolumn{4}{c}{ MODE 1 } \\
\hline $\begin{array}{c}\text { no identification } \\
\text { central }\end{array}$ & 0 & resonance & 0.0625 \\
ends of element & -0.91 & reduction & 0.0021 \\
\hline \multicolumn{4}{c}{ MODE 3 } \\
\hline no identification & 0 & resonance & 0.0019 \\
ends of element & -5.0 & reduction & 0.00013
\end{tabular}

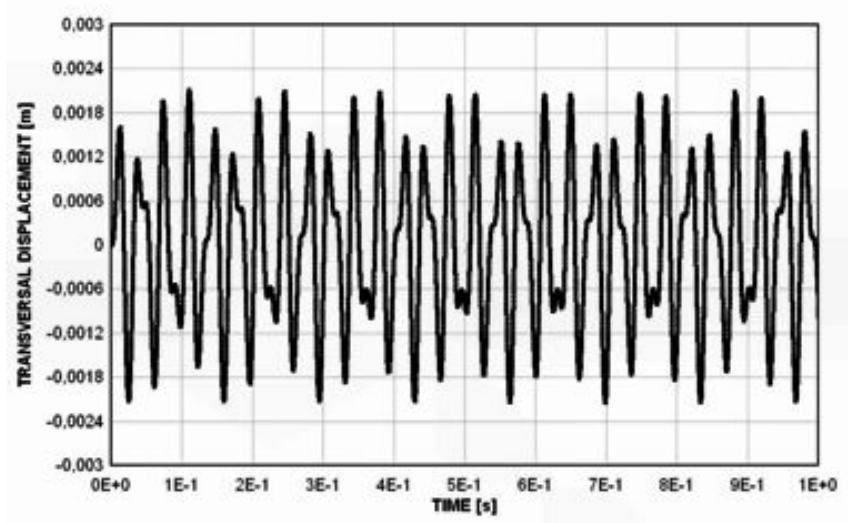

Fig. 6. The response in the middle of the beam for the first natural mode during operation of the control algorithm for moment identification in the center of the piezoelectric element.

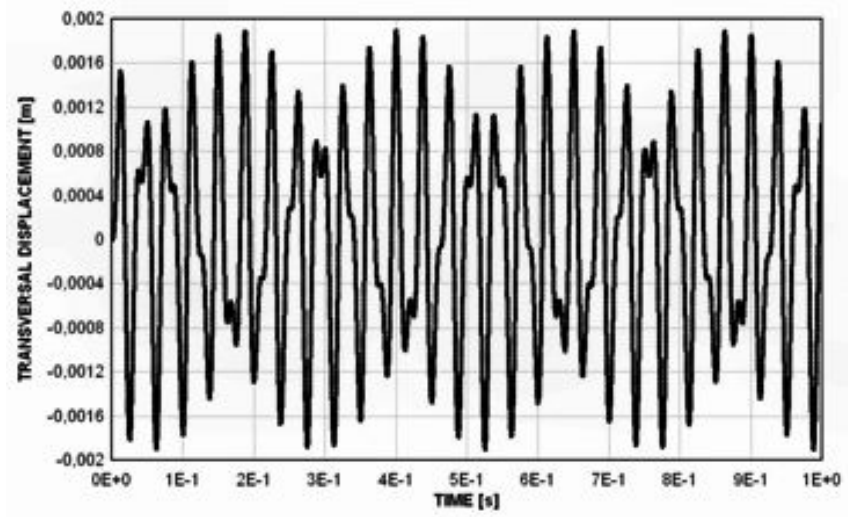

Fig. 7. The response in the middle of the beam for the first natural mode during operation of the control algorithm for moment identification at the ends of the piezoelectric element.

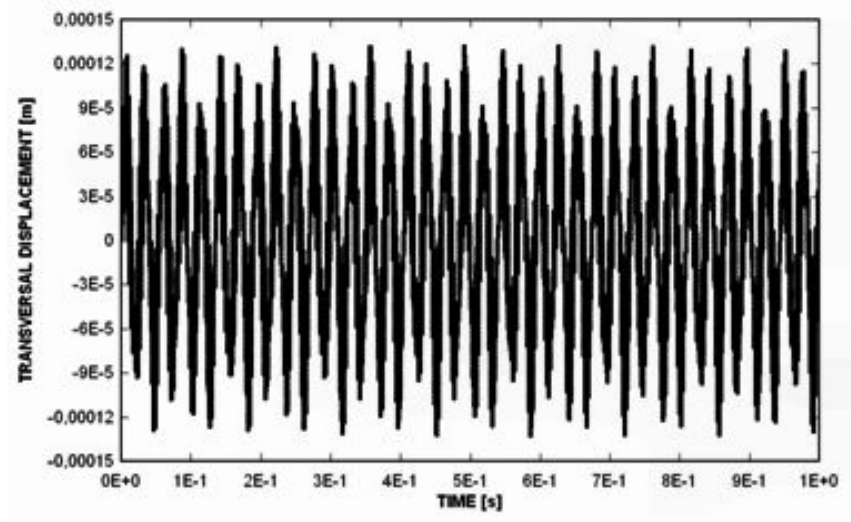

Fig. 8. The response in the middle of the beam for the third natural mode during operation of the control algorithm for moment identification at the ends of the piezoelectric element. 
The results of the analyses show that the proposed control algorithm, based on identification of the bending moment in regions of maximal curvature and applying anti-moments (in real-life, applying a voltage charge), may reduce radically the bending-excited vibrations of the beam. The proposed control algorithm must be calibrated at the beginning (identification of the feedback coefficient value).

\section{Final remarks}

The following remarks can be formulated after simulations:

- It is possible to reduce bending vibrations of beams based on the control algorithm with the assumed control parameter being the actual bending moment in the previous time step.

- The finite difference method can be applied to simulate the control of beam vibrations. Due to the applied finite difference approximation, the small difference of obtained natural frequencies can be observed. It should be taken into account in quantitative dynamic analysis.

- The model of the beam can be generalized by taking into account the material internal damping.

- It seems to be possible to apply the proposed control algorithm to multimodal response of beam vibrations. The general idea of such application is based on identification of the time-dependent bending moment value in a selected cross-section and applying the voltage charge proportional to this value to a properly positioned piezoelectric element.

\section{References}

[1] T.-T. Fu, D. Cebon, Int. J. Fatigue 22, 11 (2000).

[2] E. Baussan, J. Bielski, C. Bobeth, E. Bouquerel, O. Caretta, P. Cupial, T. Davenne, C. Densham, M. Dracos, M. Fitton, G. Gaudiot, M. Kozien, L. Lacny, B. Lepers, A. Longhin, P. Loveridge, F. Osswald, P. Poussot, M. Rooney, B. Skoczen, B. Szybinski, A. Ustrzycka, N. Vassilopoulos, D. Wilcox, A. Wroblewski, J. Wurtz, V. Zeter, M. Zito, Phys. Rev. ST - Accelerators and Beams 17, 031001 (2014).
[3] T.R. Edgecock, O. Caretta, T. Davenne, C. Densam, M. Fitton, D. Kelliher, P. Loveridge, J. Payet, M. Zito, E. Baussan, C. Bobeth, E. Bouquerel, M. Dracos, et al., Phys. Rev. ST - Accelerators and Beams 16, 021002 (2013).

[4] E. Augustyn, M.S. Kozień, Acta Phys. Pol. A 125, 164 (2013).

[5] E. Augustyn, M.S. Kozień, M. Prącik, Arch. Acoust. 39, 639 (2014).

[6] R.C. Batra, F. Dell'Isola, S. Vidoli, D. Vigilante, Int. J. Solids Struct. 42, 3115 (2005).

[7] A. Brański, Acta Phys. Pol. A 123, 1123 (2013).

[8] F. Dell'Isola, M. Porfiri, S. Vidoli, C. R. Mecanique 331, 69 (2003).

[9] U. Ferdek, M.S. Kozień, Acta Phys. Pol. A 123, 1044 (2013).

[10] C.R. Fuller, S.J. Elliot, P.A. Nielsen, Active control of vibration, Academic Press, London (1997).

[11] C.H. Hansen, S.D. Snyder, Active control of noise and vibration, E\&FN Spon, London (1997).

[12] M.S. Kozień, J. Wiciak, Acta Phys. Pol. A 118, 1186 (2010).

[13] Y. Li, J. Onoda, K. Minesugi, Acta Astronaut. 50, 335 (2002).

[14] S.O.R. Moheimani, IEEE T. Contr. Syst. T. 11, 482 (2003).

[15] D. Sun, L. Tong, Int. J. Solids Struct. 38, 3281 (2001).

[16] Ł. Ścisło, M.S. Kozień, Proceedings of the International Congress on Sound and Vibration ICSV20, Bangkok, CD (2013).

[17] G. Takacs, B. Rohal'-Illkiv, Proceedings of the Noise and Vibration: Emerging Methods NOVEM 2012, Sorrento 2012, p. 1.

[18] J. Wiciak, R. Trojanowski, Acta Phys. Pol. A 125 A179 (2014).

[19] E. Żołopa, A. Brański, Acta Phys. Pol. A 125, A155 (2014).

[20] E. Żołopa, A. Brański, Arch. Acoust. 39, 599 (2014).

[21] M.S. Kozien, Acta Phys. Pol. A 123, 1029 (2013). 